\title{
Propiedades microbiológicas, clasificación y relación estructura-actividad de cefalosporinas e importancia de las cefalosporinas de cuarta generación
}

\author{
SERGIO MELLA M..$^{1,2}$, CLAUDIA ZEMELMAN M. ${ }^{1}$, HELIA BELLO T. ${ }^{1}$, \\ MARIANA DOMINGUEZ Y. ${ }^{1}$, GERARDO GONZALEZ R. ${ }^{1}$ y RAUL ZEMELMAN Z. ${ }^{1,3 .}$ \\ MICROBIOLOGICAL PROPERTIES, CLASSIFICATIONS AND \\ STRUCTURE-ACTIVITY RELATIONSHIPS OF CEPHALOSPORINS \\ AND THE IMPORTANCE OF FOURTH GENERATION CEPHALOSPORINS
}

The cephalosporins are one of the most important types of $\beta$-lactam antibiotics. There are various classifications of these molecules, but the most frecuently used classifies them according to their structures, microbiological properties and historical development: first to fourth generation cephalosporins. Third generation cephalosporins have been extensively used, but the emergence of bacterial resistance, mainly as a consecuence of chromosomal and plasmidial production of $\beta$-lactamases has limited the use of these antibiotics. Fourth generation cephalosporins are characterized by the presence of a cuaternary nitrogen in C3; mantaining the methoxyimino aminothiazolyl in C7. Fourth generation cephalosporins display a high capacity of intracellular penetration through the external membranes of Gram negative rods and a low affinity to enzymes that degrade third generation cephalosporins. Cefepime, a fourth generation cephalosporin, has shown more inhibitory activity against chilean strains of Klebsiella pneumoniae and Escherichia coli that produce extended-spectrum $\beta$-lactamases, than cefotaxime and ceftazidime.

Key words: Cephalosporins, Cefepime, Extended-spectrum $\beta$-lactamases.

Universidad de Concepción:

Departamento de Microbiología, Facultad de Ciencias Biológicas.

Departamento de Medicina Interna, Facultad de Medicina.

Facultad de Medicina. Universidad San Sebastián, Concepción.

Este trabajo fue financiado por el Proyecto No 1980109 del Fondo Nacional de Desarrollo Científico y Tecnológico (FONDECYT) 


\section{CONSIDERACIONES GENERALES}

Las cefalosporinas son agentes antibacterianos que pertenecen al grupo de los ß-lactámicos, es decir, poseen un anillo ß-lactámico fusionado con un anillo dihidrotiazínico constituyendo el núcleo cefem del que derivan todas las cefalosporinas, a diferencia de las penicilinas que también poseen el anillo ß-lactámico pero fusionado a un anillo tiazolidínico de 5 miembros $^{1-5}$. Sobre la base de esta comparación, se puede entender que el núcleo cefem presente ventajas con relación al núcleo penam. En primer lugar, el núcleo de las cefalosporinas es intrínsecamente resistente a muchas penicilinasas; así, bacterias que producen estas enzimas permanecen susceptibles a las cefalosporinas; esta propiedad explica el amplio espectro de actividad de las cefalosporinas, particularmente sobre Staphylococcus spp. oxacilina-susceptibles $^{3}$. En segundo lugar, en el núcleo cefem son constantes la presencia de un grupo carboxílico en $\mathrm{C}_{4}$ y una cadena lateral $\mathrm{B}$-acilamínica en $\mathrm{C}_{7}$ $\left(-\mathrm{R}_{1}\right)$; sin embargo, este núcleo también permite la adición de distintos sustituyentes en $C_{3}\left(-R_{2}\right)$, a diferencia del núcleo penam donde la mayor parte de las variaciones químicas puede sólo realizarse en $\mathrm{C}_{6}\left(-\mathrm{R}^{1}\right)^{1-8}$ (Figuras 1 y 2 ).

En términos microbiológicos, estos compuestos se caracterizan por su actividad bactericida dependiente del tiempo (su máximo efecto bactericida se logra cuando en una proporción importante del intervalo interdosis la concentración plasmática del antibacteriano se encuentra

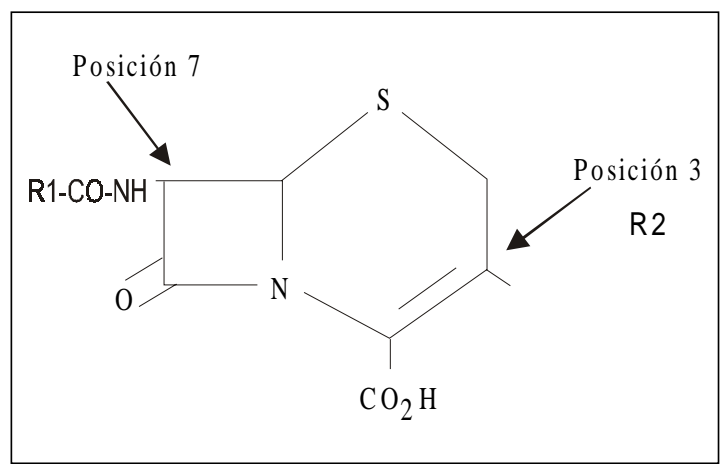

Figura 1. Núcleo cefem (núcleo básico de las cefalosporinas). sobre la CIM), del inoculo bacteriano y de la fase de crecimiento bacteriana ${ }^{7,9-13}$; amplio espectro de actividad que incluye bacterias Gram positivas, Gram negativas y anaerobios (cefoxitina, cefotetan $)^{3,6,14,15}$. Debe enfatizarse que como característica de clase las cefalosporinas no tienen actividad sobre Enterococcus spp., Staphylococcus spp. resistente a meticilina, Corynebacterium jeikeium, Stenotrophomonas maltophilia, Listeria monocytogenes, Legionella spp., Chlamydia spp. y Mycoplasma spp., 3,6,14,15. Por otra parte, dada la potente actividad bactericida, favorables parámetros farmacocinéticos, adecuada penetración a diferentes parénquimas alcanzando allí niveles bactericidas, baja toxicidad y sinergia con otros antibacterianos, las cefalosporinas son utilizadas como agentes de primera elección en el tratamiento de una serie de infecciones comunitarias y nosocomiales ${ }^{14,16-24}$.

Sin embargo, el uso creciente de esta clase de antibióticos se ha asociado en forma progresiva al aislamiento de bacterias resistentes, adquiriendo cada vez mayor importancia bacilos Gram negativos multiresistentes hiperproductores de $\beta$-lactamasas cromosomales (ß-lactamasas Amp C), destacando en este grupo Serratia, Pseudomonas, Acinetobacter, Citrobacter, Enterobacter, Morganella, Providencia y cepas de Proteus indol negativo ${ }^{25-27}$ o $\beta$-lactamasas de espectro extendido (extended spectrum $\beta$ lactamases ESBLs) de origen plasmidial, particularmente en cepas de Klebsiella pneumoniae y Escherichia coli $^{28-30}$. En este contexto, el resultado final es la selec-

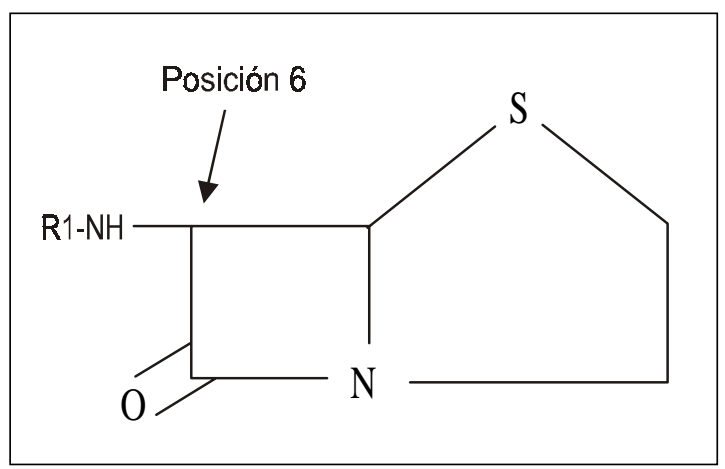

Figura 2. Núcleo penam (núcleo básico de las penicilinas). 
ción de cepas de bacilos Gram negativos capaces de degradar cefalosporinas de tercera generación (ya sea por B-lactamasas cromosomales y/o plasmidiales) lo que sumado a la capacidad de albergar otros genes de resistencia y/o alteraciones en la permeabilidad, explica la diseminación de bacterias Gram negativas multiresistentes a varias familias de antimicrobianos que incluye, entre otros, cefalosporinas de tercera generación, aminoglucósidos, quinolonas e incluso carbapenémicos ${ }^{31-35}$. Es en este marco donde se desarrollan las denominadas cefalosporinas de cuarta generación, caracterizadas fundamentalmente por mantener un amplio espectro de actividad que abarca desde Streptococcus pneumoniae, incluyendo cepas resistentes a penicilina, hasta bacilos Gram negativos multiresistentes capaces de degradar cefalosporinas de tercera generación ${ }^{25,36,37}$.

\section{Estructura y función}

El grupo activo básico de las cefalosporinas deriva de la remoción de la cadena lateral de la cefalosporina $\mathrm{C}$, producida naturalmente por Acremonium chrysogenum (antes Cephalosporium acremonium), lo que da origen al anillo 7-amino-cefalosporánico (Figura 1) ${ }^{1-6}$.

A su vez, al grupo 7-amino-cefalosporánico se le han incorporado por semisíntesis diversos grupos químicos especialmente en los carbonos 3 y 7 (radicales $R_{2}$ y $R_{1}$, respectivamente). En términos generales las sustituciones al nivel de $\mathrm{R}_{1}$ habitualmente producen moléculas con mayor afinidad por PBPs, particularmente de bacilos Gram negativos, y estabilidad frente Blactamasas, lo que clínicamente permite obtener compuestos con mayor espectro de actividad y potencia; así, el grupo 2-aminotiazolil en el carbono $7\left(\mathrm{R}_{1}\right)$ presente históricamente en moléculas como el cefotiam, permitió el desarrollo de moléculas con elevada afinidad sobre PBPs de bacterias Gram negativas, particularmente por PBPs 1A y PBP $3^{4-6,8,38}$, pero dada su inestabilidad frente a $\beta$-lactamasas, la industria farmacéutica fue capaz de desarrollar nuevas moléculas que manteniendo la capacidad de unión a PBPs fueran resistentes a la degra- dación enzimática. Así, la introducción del radical metoxi-imino sobre el carbono $\alpha$ originó las metoxi-iminocefalosporinas, las que en términos microbiológicos se caracterizan por su estabilidad frente a $\beta$-lactamasas ${ }^{4-6,8}$. Debe destacarse que uno de los grupos de moléculas de mayor uso, las cefalosporinas de tercera generación, incorporaron simultáneamente el grupo aminotiazolil y el radical metoxi-imino, lo que explica la elevada actividad de estos compuestos sobre Enterobacteriaceae $e^{4-6,8,23}$.

Por otra parte, en términos generales, las sustituciones en $R_{2}$ producen cambios en las propiedades farmacocinéticas de estas moléculas $^{4-6,8,23,39}$. Por ejemplo, el radical acetiloximetil, presente en cefalosporina $\mathrm{C}$, también se encuentra en cefalotina y cefotaxima; exhiben estos agentes una vida media muy corta, dado que son rápidamente metabolizados en el organismo por esterasas plasmáticas y hepáticas, dando origen a compuestos desacetilados que, en general, son menos activos como anti infecciosos que la molécula original ${ }^{8,40-42}$. Además, la presencia de sustituyentes pequeños y exentos de carga en $R_{2}$ es una característica general de las cefalosporinas de primera generación de uso oral; así cefalexina, cefradina y cefadroxilo, presentan como característica un grupo metilo a este nivel, interesantemente cefaclor presenta un cloro en $\mathrm{R}_{2}^{4-6,16,41}$. Por otra parte, $\mathrm{y}$ también desde una perspectiva general, las cefalosporinas de uso parenteral tienden a presentar vidas medias más prolongadas en relación a la presencia de radicales acídicos y de mayor tamaño en $\mathrm{R}_{2}$, como en ceftriaxona que presenta un anillo heterocíclico de carácter ácido (1,2,5,6 - tetrahidro- 2-metil 5,6-dioxo- 1,2,4 - triazin -3-il) que permite explicar niveles plasmáticos más elevados y tiempo de vida media más prolongado que el resto de las aminotiazolil metoxi-imino cefalosporinas $^{39,41,43}$. Otro ejemplo al respecto es la comparación entre cefazolina (cefalosporina con un anillo heterocíclico en $\mathrm{R}_{2}$ ) y cefalotina (acetiloximetilo cefalosporina), presentando las primeras concentraciones plasmáticas más elevadas y vida media más prolongada que la segunda ${ }^{5,8,41}$. Otro grupo de importancia en este sustituyente es el radical N-metiltetrazol, presente en: moxalactam, cefamandol, cefoperazona 
cefmenoxima, cefpiramida, cefmetazol, cefotetan y cefbuperazona, que explica la presencia de efectos adversos con el uso de estas moléculas, principalmente de tipo hematológico con prolongación del tiempo de protrombina y efecto "de tipo disulfiram", por alteración en la síntesis de vitamina $\mathrm{K}$ en el intestino e inhibición del metabolismo del alcohol, respectivamente ${ }^{3,5-7,23}$. Finalmente, otro grupo interesante a este nivel es el radical 3'mercaptotiazida, presente en cefodizima, cefalosporina con favorables parámetros farmacocinéticos y una peculiar actividad inmunomoduladora ${ }^{44}$.

También, desde una perspectiva histórica, un grupo muy interesante de moléculas en que se utilizaron sustituyentes catecólicos en $\mathrm{R}_{1} \mathrm{y}$ en menor medida en $\mathrm{R}_{2}$, fueron las denominadas cefalosporinas catecólicas, que presentaban una favorable penetración intracelular en bacilos Gram negativos, ya que eran activamente transportadas a través de proteínas de membrana externa reguladas por fierro (IROMPs) $^{45-47}$.

Desde esta misma perspectiva, en el intento de lograr moléculas con propiedades microbiológicas y/o farmacocinéticas superiores, se sintetizaron compuestos experimentales cuya estructura química correspondía a la asociación de una cefalosporina con una quinolona, las así denominadas moléculas duales, destacando en este grupo Ro 24-6392, molécula cuya estructura química derivaba de la asociación entre desacetilcefotaxima y una sustitución 3' con ciprofloxacina y que exhibía una actividad antimicrobiana comparable a la de ciprofloxacina sobre bacterias Gram negativas y a la de cefotaxima sobre bacterias Gram positivas ${ }^{47}$.

En términos generales, la, adición de nuevos sustituyentes permite:

- Ampliar el espectro de actividad

- Mejorar las características farmacocinéticas

- Ampliar la actividad a bacterias de difícil tratamiento como Pseudomonas spp, Citrobacter spp, Serratia spp, Enterobacter spp, etc.

- Aumentar la estabilidad frente a la hidrólisis de B-lactamasas

- Describir nuevas propiedades biológicas como la inmunomodulación

\section{Mecanismo de acción}

Las cefalosporinas, al igual que el resto de los antibióticos ß-lactámicos, ejercen su actividad antibacteriana inhibiendo la síntesis del peptidoglicano, produciendo finalmente lisis bacteriana $^{3,7,9,23}$. El mecanismo de acción deriva de la unión covalente del ß-lactámico al sitio activo de las enzimas denominadas PBPs. Esta reacción se explica porque los ß-lactámicos poseen una estructura química similar a los dos últimos aminoácidos del pentapéptido (D-alanina-D-alanina) que une las moléculas de peptidoglicano. Por otra parte, las PBPs ejercen actividad bioquímica de transglicosilasas (complejo PBP1), transpeptidasas (PBP3) y carboxipeptidasas (PBP4, 5 y 6); además, este grupo de antimicrobianos produce una activación de autolisinas bacterianas que destruyen el peptidoglicano ${ }^{5,49-51}$. La activación de la actividad autolítica de la bacteria ha ido adquiriendo mayor relevancia por la relación que se establecería entre el desarrollo de tolerancia y la ausencia de activación de esta vía ${ }^{51}$. Clásicamente se ha descrito que con la unión de la cefalosporina a la PBP se bloquea la actividad de transglicosilasa o transpeptidasa interrumpiéndose el ensamblaje de las moléculas precursoras del peptidoglicano (N-acetilmurámico + péptido y $\mathrm{N}$-acetilglucosamina en bacilos Gram negativos o incluyendo un pentapéptido de glicina en $S$. aureus), de esta manera se interrumpe la síntesis de esta cubierta. Esto sumado a la activación autolítica llevaría finalmente a la lisis osmótica y por tanto a la muerte de la bacte$\mathrm{ria}^{6,7,49,51}$.

En las bacterias Gram negativas las PBPs se disponen hacia el espacio periplásmico por la presencia de la membrana externa (que constituye una barrera al paso de una importante cantidad de moléculas); a diferencia de las bacterias Gram positivas, donde las PBPs se encuentran en la membrana citoplasmática expuestas al medio externo ${ }^{52}$. Por tanto, para que una cefalosporina alcance su blanco (PBP) en un bacilo Gram negativo debe poseer una adecuada penetración a través de las porinas de la membrana externa (canales que permiten el ingreso de moléculas desde el medio externo hacia el espacio periplásmico). En este contex- 
to, la mayor penetración intracelular es una característica microbiológica de interés de las cefalosporinas de cuarta generación ${ }^{36,53}$.

\section{Clasificación}

Existe un número importante de clasificaciones, destaca entre ellas la clasificación biológica de O'Callaghan, actualmente de carácter histórico, que subdividió a estos antimicrobianos en 7 grupos de acuerdo a su metabolismo y estabilidad a la degradación por ß-lactamasas ${ }^{54}$. Otra clasificación, de carácter químico, agrupa a las cefalosporinas de acuerdo a los sustituyentes presentes en el carbono de la cadena lateral de $\mathrm{C}_{7}$, distinguiéndose cuatro grupos: cefalosporinas sustituidas y no sustituidas a este nivel; $\alpha$ - amino cefalosporinas y aril-oxi-imino cefalosporinas ${ }^{44}$. Interesantemente, otra clasificación es sólo de carácter farmacocinético, de acuerdo al tiempo de vida media, y aplicada fundamentalmente a las cefalosporinas de uso parenteral, así en el grupo 1, se incluyen aquellos compuestos que tienen vidas medias menores de 1 hora (cefalotina, cefradina, cefapirina, cefoxitina); en el grupo 2 compuestos con vidas medias entre 1 y 3 horas (cefazolina, cefmetazol, cefuroxima, cefotaxima, ceftixozima, moxalactam, cefmenoxima, cefoperazona, ceftazidima, cefpiroma, cefepime) y finalmente en el grupo 3 aquellas cefalosporinas con tiempos de vida media mayores a 3 horas (cefpiramide, ceftriaxona, cefodizima $)^{23,55}$. A su vez, dentro de cada uno de estos grupos las moléculas son subdivididas según la ruta principal de eliminación (renal, biliar). Otras clasificaciones subdividen a las cefalosporinas de acuerdo a sus propiedades microbiológicas, principalmente el espectro de actividad. En los grupos I y II, cefalosporinas de amplio espectro, se incluyen moléculas de primera y segunda generación, respectivamente. En el grupo III, se incluyen moléculas de tercera generación y oxacefems. En el grupo IV cefalosporinas de cuarta generación. En el grupo V, o de cefalosporinas de reducido espectro, se incluye a cefsulodina ${ }^{44}$.

Otra clasificación, más funcional en una perspectiva microbiológica, fue desarrollada por J.D. Williams, quien agrupó a estas moléculas de acuerdo a su espectro antibacteriano enfatizando la cobertura sobre bacterias Gram positivas, Gram negativas, Pseudomonas aeruginosa y anaerobias. Así el grupo I incluye a las moléculas con mayor actividad sobre Gram positivos (cefradina, cefradoxilo, cefalexina, cefalotina); el grupo II, a cefalosporinas con mayor actividad sobre Gram negativos (cefuroxima, cefotaxima, ceftriaxona, ceftizoxima); el grupo III a compuestos con actividad sobre $P$. aeruginosa (ceftazidima, cefoperazona) y el grupo IV a moléculas con actividad sobre bacterias anaerobias (cefoxitina, cefotetan, moxalactam $)^{56}$.

Sin embargo, desde el punto de vista clínico, la clasificación más utilizada agrupa a estos compuestos de acuerdo al desarrollo histórico y algunas características microbiológicas y estructurales en común, las denominadas generaciones de cefalosporinas ${ }^{3,4-6,14}$. A las ya conocidas moléculas de primera, segunda y tercera generación se agregan las denominadas cefalosporinas de cuarta generación, destacando en este grupo: cefepime y cefepiroma, aunque también se incluyen en este grupo otras moléculas todavía en fase de desarrollo como: cefoselis, cefclidin, cefozopram y cefluprenam ${ }^{57-62}$.

Dado que la clasificación en generaciones es la de mayor uso, se discutirán algunas características relevantes de éstas:

Las cefalosporinas de primera generación presentan mejor actividad sobre cocos Gram positivos como $S$. aureus susceptible a meticilina, $S$. pyogenes y $S$. pneumoniae; $\sin$ embargo, como ya se comentó, carecen de actividad sobre Enterococcus spp. Su actividad sobre bacilos Gram negativos es limitada a cepas de E. coli, Klebsiella spp. y Proteus mirabilis (no productoras de B-lactamasas cromosomales y/o plasmidiales) $)^{3,7,14}$. A pesar de la descripción de actividad in vitro sobre algunas cepas de Salmonella y Shigella, las moléculas de este grupo no son útiles para el tratamiento de infecciones producidas por estos patógenos ${ }^{63-65}$. Además, carecen de una actividad significativa sobre Haemophilus influenzae $e^{3,7,14,23}$. En este grupo existen moléculas de uso oral y parenteral; desde un punto de vista farmacológico se caracterizan por sus cortos tiempos de vida media (aunque cefazolina 
y cefadroxilo presentan tiempos de eliminación más prolongados que el resto de las moléculas del grupo), baja penetración al LCR y excreción por vía urinaria alcanzando adecuados niveles terapéuticos para el tratamiento de infecciones por especies uropatógenas comunitarias susceptibles ${ }^{3,16,23,66}$.

La característica microbiológica definitiva de las cefalosporinas de segunda generación es su actividad sobre $H$. influenzae, Moraxella catarrhalis, Neisseria meningitidis y Neisseria gonorrhoeae. En esta generación de cefalosporinas, también se incluyen otras moléculas que técnicamente corresponden a:

- Cefamicinas: químicamente son 7-alfa metoxi-cefalosporinas. Estas moléculas cefoxitina, cefotetan- presentan una potente actividad sobre Bacteroides fragilis $s^{3,67,68}$.

- Carbacefems: químicamente estos compuestos se caracterizan porque el azufre del anillo dihidrotiazínico del grupo cefem está sustituido por un carbono (carba) de un grupo metileno $\left(\mathrm{CH}_{2}\right)$. Así loracarbef se diferencia de cefaclor sólo por el cambio estructural previamente señalado (carbono en vez de azufre $)^{69}$.

Por otra parte, la descripción de una mayor actividad microbiológica de moléculas de este grupo sobre enterobacterias resistentes a cefalotina - como cepas de Enterobacter, Serratia, Citrobacter, Providencia y Proteus indol-positivos - carece de importancia microbiológica y clínica, dados los actuales perfiles de susceptibilidad de los bacilos Gram negativos ${ }^{26,30,70,71}$.

Debe enfatizarse que los antimicrobianos de esta generación - incluyendo tanto a cefalosporinas, cefamicinas y carbacefems (o carbacefemas)carecen de actividad sobre $P$. aeruginosa; además, con la excepción de cefamandol, son menos activos que sus congéneres de primera generación sobre cepas de $S$. aureus susceptibles a oxacilina ${ }^{3,5,23,72}$. Desde un punto de vista farmacológico no difieren en forma sustancial de las moléculas de primera generación, excepto en su mejor penetración al LCR; sin embargo, no se consideran agentes de elección en el tratamiento de MBA debido a la menor eficacia clínica al compararlas con moléculas de tercera generación, por ejemplo cefuroxima versus ceftriaxona ${ }^{20,73}$.

Las cefalosporinas de tercera generación constituyen uno de los grupos de antimicrobianos de mayor uso en la actualidad. Los compuestos de esta generación se caracterizan por presentar, al menos, dos de las siguientes características:

- en C-7 del núcleo cefem, la presencia de un anillo 2-aminotiazolil como cadena lateral

- amplio espectro de actividad

- elevada actividad sobre Enterobacteriaceae (excepto sobre cepas hiperproductoras de cefalosporinasas y/o ESBLs), $H$. influenzae, Neisseria spp., S. pyogenes y $S$. pneumoniae y actividad sobre $P$. aeruginosa (ceftazidima y cefoperazona $)^{42,74}$.

En este contexto es importante destacar que si bien ceftriaxona y cefotaxima son agentes de elección en el tratamiento de MBA adquirida en la comunidad, la descripción creciente de cepas de $S$. pneumoniae resistentes a penicilina y cefalosporinas ha limitado en algunos centros, su uso como monoterapia empírica para el tratamiento de infecciones meníngeas ${ }^{75}$. Por otra parte, la actividad de estos agentes sobre $S$. aureus susceptible a meticilina es menor que la de los congéneres de primera y segunda generación, debiéndose considerar que ceftazidima y los agentes de uso oral cefixima y ceftibuten carecen de una actividad microbiológica útil sobre este patógeno ${ }^{7,23,76,77}$. Desde una perspectiva farmacológica en este grupo existen moléculas como ceftriaxona que presentan tiempos de vida media prolongados, permitiendo su dosificación cada 12 o incluso cada 24 horas; además, cefotaxima es metabolizada a una forma biológicamente activa (desacetilcefotaxima) y por otra parte estos agentes alcanzan concentraciones útiles en una serie de tejidos y parénquimas, entre ellos sangre, orina, bilis (particularmente cefoperazona y ceftriaxona), pulmones, líquido peritoneal y meninges ${ }^{3,14,39,78}$.

Es interesante destacar que en los grupos analizados, cefalosporinas de primera hasta tercera generación, hay agentes de uso oral, que en términos generales mantienen las características microbiológicas generales de las respectivas generaciones, por ejemplo, cefadroxilo, cefuroxima y cefixima, respectivamente ${ }^{7,16,77}$. 


\section{CEFALOSPORINAS DE CUARTA GENERACION}

Las cefalosporinas de cuarta generación se caracterizan químicamente por la presencia de un grupo metoxi-imino aminotiazolil en $\mathrm{R}_{1}$ del núcleo cefem (similar al que presentan las moléculas de tercera generación), excepto cefclidin (previamente E 1040) que presenta un radical muy similar: aminotiadiazolil, y principalmente por la presencia de un nitrógeno cuaternario en $\mathrm{R}_{2}$ (Figura 3). Este grupo se mantiene cargado positivamente tanto en condiciones de $\mathrm{pH}$ ácido como alcalino. La asociación de esta carga neta positiva con una carga negativa en $\mathrm{C}_{4}$ del núcleo cefem hace de estos antibacterianos moléculas zwitteriónicas, es decir, moléculas con un balance entre sus cargas positivas y negativas en los rangos de $\mathrm{pH}$ encontrados in vivo (Figura 4) $36,53,57,58,79$.

Esta característica químico-estructural explica por qué estas cefalosporinas presentan una penetración a través de las porinas, superior a sus congéneres de tercera generación, lo que le permite alcanzar altas concentraciones en el espacio periplásmico de bacilos Gram negativos ${ }^{79,80}$. Experimentalmente se ha demostrado que cefpiroma, cefepima y cefclidin tienen una velocidad de penetración intracelular, sobre cepas de E. coli y E. cloacae, varias veces superior a la de cefotaxima y ceftazidi$\mathrm{ma}^{79-81}$. Esta propiedad se relacionaría con el balance de cargas químicas de estos compuestos, ya que, si bien las cefalosporinas de cuarta generación son moléculas de mayor tamaño que sus congéneres de tercera generación, presentan en su estructura química una importante carga positiva (a nivel del amonio cuaternario) y además son selectivamente incorporadas a través de la porina Omp F $F^{36,58,79-81}$. Ceftazidima, en cambio, tiene una carga neta negativa, a pesar de presentar una carga positiva en $\mathrm{C}_{3}$. $\mathrm{La}$ penetración intracelular más lenta de esta molécula puede explicarse entonces por el potencial de Donnan de la bacteria (interior negativo) ${ }^{58,80}$. Sin embargo, el solo aumento de la penetración intracelular, no explica en forma exclusiva la importante actividad antibacteriana de estos compuestos, particularmente sobre bacterias resistentes. Así, cefaloridina que estruc-

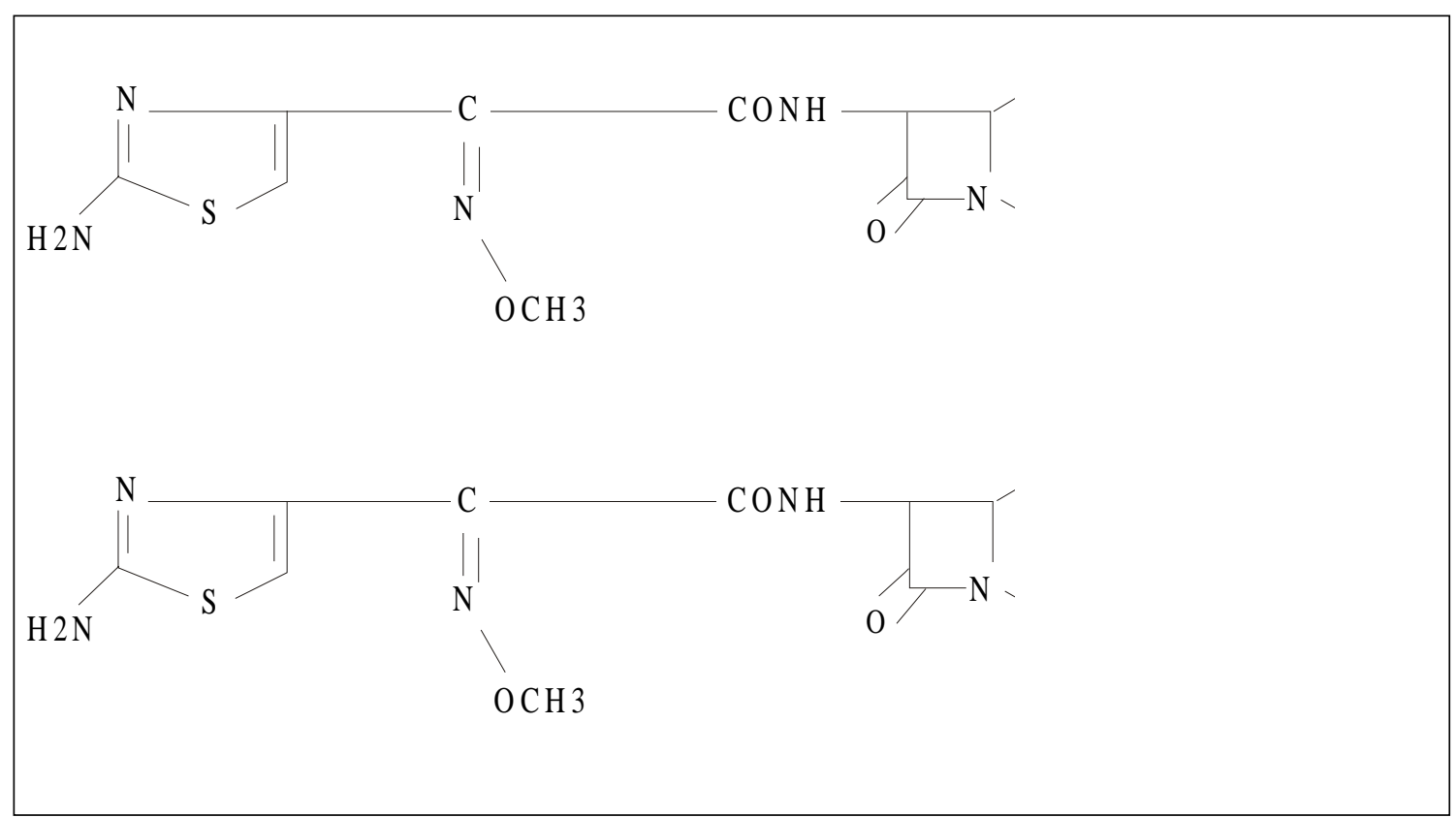

Figura 3. Estructura química de cefepime y cefpirome, respectivamente. 


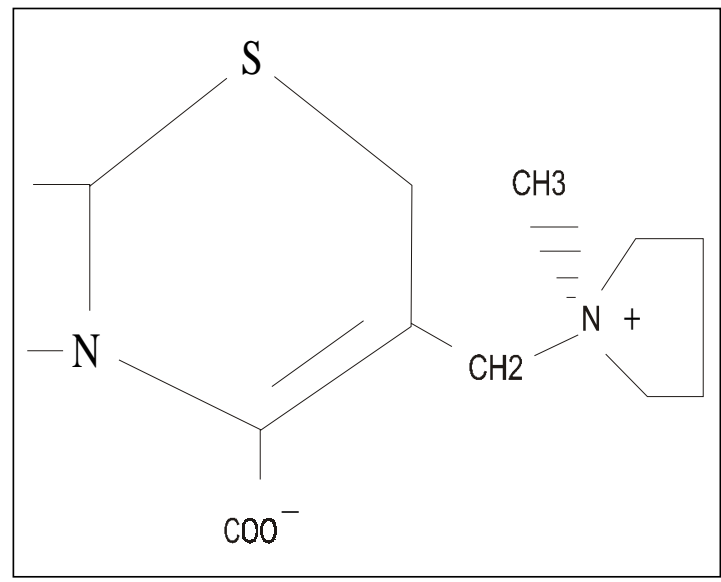

Figura 4. Estructura zwitteriónica de las cefalosporinas de cuarta generación.

turalmente también es una molécula dipolar, sin carga neta, con un amonio cuaternario en $\mathrm{C}_{3}$, presenta una penetración intracelular incluso superior a las moléculas de cuarta generación; sin embargo, al carecer del radical metoxiimino no presenta una adecuada estabilidad frente a beta-lactamasas, siendo rápidamente degradada ${ }^{5,36,58}$.

Por lo anterior otro aspecto relevante para explicar la mayor actividad de estas cefalosporinas sobre bacterias Gram negativas resistentes a moléculas de tercera generación, es la estabilidad frente a las ß-lactamasas tanto cromosomales como plasmidiales y su afinidad por el sitio blanco: PBP's $\mathrm{s}^{36,58,80,82,83}$. Así, la actividad sobre cepas hiperproductoras de B-lactamasas del grupo 1 de Bush (cefalosporinasas cromosomales) se explica por la baja afinidad de la ß-lactamasa por la cefalosporina (en general valores más elevados para $\mathrm{K}_{\mathrm{m}}$ ); habiéndose establecido que la afinidad de estas enzimas por cefotaxima es 1.000 a 10.000 veces mayor que sobre cefepima y del orden de 10 a 100 veces superior para ceftazidima en comparación con cefepima ${ }^{36,58}$.

Por otra parte, el efecto final de los sustituyentes en la relación estructura-actividad de estos compuestos, es decir, mantención de sustituyentes con importante actividad sobre Gram negativos como metoxi-imino, como la presencia en la misma molécula de radicales que incrementan la actividad sobre cocáceas Gram positivas como amonio cuaternario, explican la actividad microbiológica "más balanceada" de estos antimicrobianos, ya que presentan una elevada actividad in vitro sobre Gram positivos, particularmente, S. pneumonaie (incluyendo cepas resistentes a penicilina), junto a una potente actividad inhibitoria y bactericida sobre bacilos Gram negativos entéricos, incluyéndose, como ya se mencionó, cepas multiresistentes capaces de degradar moléculas de tercera generación (Tabla 1) ${ }^{36,84-86}$.

En forma general y práctica puede señalarse que estos agentes despliegan una actividad superior a la de ceftriaxona y cefotaxima sobre bacilos Gram negativos multiresistentes (Serratia, Citrobacter, Enterobacter, Morganella, Providencia y cepas de Proteus indol negativo hiperproductoras de cefalosporinasas y/o sobre de cepas de $K$. pneumoniae y E. coli productoras de ESßLs ) y equivalente al de estos compuestos de tercera generación sobre cepas de bacterias Gram negativos susceptibles y S. pneumoniae ${ }^{36,58,84-86}$. Con relación a su actividad sobre $P$. aeruginosa, estas moléculas tienen una actividad antibacteriana comparable, pero no superior, a la de ceftazidima ${ }^{36,53}$, $57,58,82,83$.

En este contexto, en nuestro laboratorio evaluamos la actividad de cefepima, cefotaxima y ceftazidima sobre cepas de $K$. pneumoniae y E. coli productoras de ESBLs aisladas de diferentes hospitales chilenos. Es interesante destacar que de las cepas originalmente recibidas en nuestro estudio, se determinó la producción de ESBLs en $43,9 \%$ de las cepas de $K$. pneumoniae y en $8 \%$ de las cepas de E. coli. Cefepima demostró ser el ß-lactámico de mayor actividad sobre las cepas ensayadas. Así, $2,6 \%$ de los aislamientos de $K$. pneumoniae y $25 \%$ de las cepas de $E$. coli fueron resistentes a este agente. Al analizar la Tabla 1 puede comprobarse también que con la asociación con ácido clavulánico se produce un importante incremento en la susceptibilidad de las bacterias en estudio a las cefalosporinas de tercera generación. Este efecto fue más pronunciado sobre $K$. pneumoniae con disminución de las frecuencias de resistencia de $100 \%$ (cefalosporina) a $4 \%$ (cefalosporina asociada a inhibidor 
Tabla 1. Actividad inihibitoria de cefepime, cefotaxima y ceftazidima, en presencia y ausencia de ácido clavulánico, sobre 75 cepas de $K$. pneumoniae y 12 cepas de $E$. coli productoras de ESBLs aisladas de diferentes hospitales chilenos

\begin{tabular}{lllcrc}
\hline \multirow{2}{*}{ Antibiótico } & Microorganismo & \multicolumn{1}{c}{ Rango } & $\mathbf{5 0}$ & $\mathbf{9 0}$ & \% resistencia \\
\hline CFTX & K. pneumoniae & $4-256$ & 32 & 64 & 100 \\
& E. coli & $4-256$ & 64 & 128 & 92 \\
CFTX + AC & K. pneumoniae & $<0,25-2$ & $<0,25$ & 16 & 3 \\
& E. coli & $1-32$ & 2 & 32 & 25 \\
CFZD & K. pneumoniae & $8->256$ & 32 & $>256$ & 100 \\
& E. coli & $8->256$ & 64 & 256 & 100 \\
CFZD + AC & K. pneumoniae & $<0,25-16$ & 1 & 4 & 4 \\
& E. coli & $2->32$ & 4 & 32 & 25 \\
CFPM & K. pneumoniae & $<0,25-128$ & $<0,25$ & 16 & 2,6 \\
& E. coli & $<0,25-256$ & $<0,25$ & 32 & 25 \\
CFPM + AC & K. pneumoniae & $<0,25-32$ & $<0,25$ & 2 & 0 \\
& E. coli & $<0,25-32$ & $<0,25$ & 32 & 17 \\
\hline
\end{tabular}

Cefotaxima (CFTX), Ceftazidima (CFZD), Cefepima (CFPM), Acido clavulánico (AC)

*Cefalosporina: ácido clavulánico, 2:1

de B-lactamasa). La mayor susceptibilidad de las bacterias a la asociación de cefalosporina de tercera generación y ácido clavulánico (AC) se explica por la actividad hidrolizante de ESßLs sobre las cefalosporinas. Por lo tanto, el escaso efecto sinergístico de AC y cefepima sugiere una escasa actividad de ESßLs como mecanismo de resistencia a esta cefalosporina de cuarta generación. De lo anterior puede concluirse que cefepima es activa sobre una importante proporción de cepas chilenas de K. pneumoniae y de E. coli productoras de ESßLs.

Como última característica microbiológica general, debe destacarse que estos compuestos son débiles inductores de la producción de ß-lactamasas cromosomales, a diferencia de lo que ocurre con carbapenémicos y cefamicinas, que característicamente son potentes inductores de B-lactamasas Amp C $\mathrm{C}^{25,37}$.

Finalmente, basándonos en el aumento cada vez más creciente de la resistencia bacteriana, la mayor sobrevida de huéspedes susceptibles, el lento desarrollo de nuevas familias de antimi- crobianos (con excepción de las oxazolidinonas) y las propiedades microbiológicas de las cefalosporinas de cuarta generación, debemos plantear que -de acuerdo a los estudios de susceptibilidad locales- las cefalosporinas de cuarta generación podrían representar una alternativa terapéutica para el tratamiento de infecciones producidas por bacilos Gram negativos resistentes a cefalosporinas de tercera generación ${ }^{87-91}$.

\section{RESUMEN}

Las cefalosporinas son uno de los grupos de mayor importancia dentro de los B-lactámicos. Existen diversas clasificaciones de estas moléculas, siendo la más utilizada aquella que agrupa a estos compuestos de acuerdo a propiedades estructurales, microbiológicas y desarrollo histórico: primera a cuarta generación. Las cefalosporinas de tercera generación han sido ampliamente utilizadas, pero la emergencia de 
resistencia bacteriana fundamentalmente derivada de la producción de B-lactamasas tanto cromosomales como plasmidiales, ha limitado el uso de estos compuestos. Las cefalosporinas de cuarta generación se caracterizan por la presencia de un nitrógeno cuaternario en $\mathrm{C}_{3}$, además de mantener el grupo metoxi-imino aminotiazolil en $\mathrm{C}_{7}$. Presentan una elevada penetración intracelular a través de la membrana externa de bacilos Gram negativos y tienen una baja afinidad por enzimas que degradan cefalosporinas de tercera generación. Cefepime, una cefalosporina de cuarta generación, demostró una mayor actividad inhibitoria sobre cepas chilenas de Klebsiella pneumoniae y Escherichia coli productoras de $\beta$-lactamasas de espectro extendido, que cefotaxima y ceftazidima.

\section{BIBLIOGRAFIA}

1.- ROLINSON G N. The influence of 6-aminopenicillanic acid on antibiotic development. J Antimicrob Chemother 1988; 22: 5-14.

2.- ZEMELMAN R, NORAMBUENA R, VERGARA L, GACITUA R. Los antibióticos ß-lactámicos: agrupación según su estructura química y sus propiedades bacteriológicas. Rev Méd Chile 1987; 115: 983 91.

3.- FRIED J S, HINTHORN D R. The cephalosporins. Dis a Month 1985; 31: 1-60.

4.- NEU HC. Structure-activity relations of new B-lactam compounds and in vitro activity against common bacteria. Rev Infect Dis 1983; 5 (suppl 2): S319-.

5.- NEU H C. Beta-lactam antibiotics structural relationships affecting in vitro activity and pharmacological properties. Rev Infect Dis 1986; 8 (Suppl 3): 237-59

6.- NEU H C. Relation of structural properties of betalactam antibiotics to antibacterial activity. Am J Med 1985; 79 (Suppl 2 A): 2-13.

7.- SCHOLAR E M, PRATT W B (Eds). The inhibitors of cell wall synthesis, II. The antimicrobial drugs. Oxford University Press. 2000 Nueva York. pp:

8.- LOPEZ F, GARRIDO J C. Química y relación estructura-actividad de los antibióticos cefalosporínicos (I). Rev Col Químico Farmacéutico 1988; 44: 110-22.

9.- TURNIDGE J D. The pharmacodynamics of B-lactams. Clin Infect Dis 1998; 27: 10-22.

10.- CRAIG W A. The future-can we learn from the past? Diagn Microbiol Infect Dis 1997; 27: 49-53.

11.- DUDLEY M N. Pharmacodynamics and pharmacokinetics of antibiotics with special reference to the fluoroquinolones. Am J Med 1991; 91 (Suppl $6 \mathrm{~A}): 6 \mathrm{~A}-45 \mathrm{~S}$.

12.- NICOLAU D P, QUINTILIANI R, NIGHTINGALE
C H. Cinética y dinámica de los antibióticos para el clínico. Clin Med Nort Am 1995; 79: 483-500.

13.- DRUSANO G L. Human pharmacodynamics of betalactams, aminoglycosides and their combination. Scand J Infect Dis 1991 (Suppl); 74: 235-48.

14.- MARSHALL W F, BLAIR J E. The cephalosporins. Mayo Clinic Proc 1999; 74: 187-95.

15.- DONOWIRZ G R, MANDELL G L. Beta-lactam antibiotics. N Engl J Med 1988; 318: 490-500.

16.- PAYA E. Ventajas y desventajas de las cefalosporinas orales de segunda y tercera generación. Rev Chil Infect 1997; 14: 233-8.

17.- AMERICAN THORACIC SOCIETY. Guidelines for the initial management of adults with communityacquired pneumonia: diagnosis, assessment of severity, and initial antimicrobial therapy. Am Rev Respir Dis 1993; 148: 1418-26.

18.- BARTLETT J G, BREIMAN R F, MANDELL L A, FILE T M Jr. Community-acquired pneumonia in adults: guidelines for management. The Infectious Diseases Society of America. Clin Infect Dis 1998: 26: 811-38.

19.- MARRIE T J. Community-acquired pneumonia: epidemiology, etiology, treatment. Infect Dis Clin North Am 1998; 12: 723-40

20.- TUNKEL A R, SCHELD W M. Acute meningitis. In: Mandell, Douglas \& Bennett's. Principles and Practice of Infectious Diseases. Mandell G L, Bennett J E, Dolin R, eds. Fifth edition. Philadelphia: Editorial Churchill Livingstone 2000; 959-96.

21.- AMERICAN THORACIC SOCIETY. Hospitalacquired pneumonia in adults: diagnosis, assessment of severity, initial antimicrobial therapy, and preventive strategies. A consensus statement. Am J Respir Crit Care Med 1996; 153: 1711-25.

22.- BARTLETT J G, DOWELL S F, MANDELL L A, FILE T M, MUSHER D M, FINE M J. Practice guidelines for the management of community-acquired pneumonia in adults. Clin Infect Dis. 2000; 31: 347 82

23.- KARCHMER A W. Cephalosporins. In: Mandell, Douglas \& Bennett's. Principles and Practice of Infectious Diseases. Mandell G L, Bennett J E, Dolin R, eds. Fifth edition. Philadelphia: Churchill Livingstone 2000; 274-99.

24.- ADU A, ARMOUR C L. Drug utilisation review (DUR) of the third generation cephalosporins. Drugs 1995; 50: 423-39.

25.- JONES R N. Important and emerging B-lactamase mediated resistances in hospital-based pathogens: the Amp C enzymes. Diagn Microbiol Infect Dis 1998; 31: 461-6.

26.- JARVIS W R, MARTONE W J. Predominant pathogens in hospital infections. J Antimicrob Chemother 1992; 29: 19-24.

27.- LIVERMORE D M. ß-lactamases in laboratory and clinical resistance. Clin Microbiol Rev 1995; 8: 557 84.

28.- HERITAGE J, M'ZALI F H, GASCOYNE-BINZI D, HAWKEY P M. Evolution and spread of SHV 
extended-spectrum 3 -lactamases in gram negative bacteria. J Antimicrob Chemother 1999; 44: 309-18.

29.- PODSCHUN R, ULLMAN U. Klebsiella spp. as nosocomial pathogens: epidemiology, taxonomy, typing methods, and pathogenicity factors. Clin Microbiol Rev 1998; 11: 589-603.

30.- PITOUT J D D, SANDERS C C, SANDERS W E. Antimicrobial resistance with focus on ß-lactam resistance in Gram negative bacilli. Am J Med 1997; 103: 51-9.

31.- HANBERGER H, GARCIA-RODRIGUEZ J A, GOBERNADO et al. Antibiotic susceptibility among aerobic Gram negative bacilli in intensive care units in 5 european countries. JAMA 1999; 281: 67-71.

32.- CHARREL R N, PAGES J M, DE MICCO P et al. Prevalence of outer membrane porin alteration in $B$ lactam-antibiotic-resistant Enterobacter aerogenes. Antimicrob Agents Chemother 1996; 40: 2854-8.

33.- BRADFORD P A, URBAN C, MARIANO N, PROJAN S J, RAHAL J J, BUSH K. Imipenem resistance in Klebsiella pneumoniae is associated with the combination of ACT-1, a plasmid-mediated Amp C B-lactamase, and the loss of an outer membrane protein. Antimicrob Agents Chemother 1997; 41: 563-9.

34.- QUEENAN A M, TORRES-VIERA C, GOLD H et al. SME-type carbapenem-hydrolizing class A Blactamases from geographically diverse Serratia marcescens strains. Antimicrob Agents Chemother 2000; 44: 3035-9.

35.- COHEN S P, MC MURRY L M, HOOPER D C, WOLFSON J S, LEVY S B. Cross-resistance to fluoroquinolones in multiple-antibiotic-resistance (Mar) Escherichia coli selected by tetracycline resistance: decreased drug accumulation asociated with membrane changes in addition to Omp $\mathrm{F}$ reduction. Antimicrob Agents Chemother 1989; 33:1318-25.

36.- SANDERS C C. Cefepime: The next generation? Clin Infect Dis 1993; 17: 369-79

37.- GARAU J. The clinical potential of fourth-generation cephalosporins. Diagn Microbiol Infect Dis 1998; 31: 479-80.

38.- CURTIS N A C, ORR D C, ROSS G W, BOULTON M G. Affinities of penicillins and cephalosporins for the penicillin-binding proteins of Escherichia coli K-12 and their antibacterial activity. Antimicrob Agents Chemother 1979; 16: 533-9.

39.- BALANT L, DAYER P, AUCKENTHALER R. Clinical pharmacokinetics of the third generation cephalosporins. Clin Pharmacokinetics 1985; 10: 10143.

40.- WHEELER W J. Cephalosporins and other betalactam antibiotics. Lloydia. 1977; 40: 519-42.

41.- LOPEZ F, GARRIDO J C. Química y relación estructura-actividad de los antibióticos cefalosporínicos. Rev Col Químico Farmacéutico 1989; 41: 5-14.

42.- FU K P, ASWAPOKEE P, HO I, MATTHIJSSEN C, NEU H C. Pharmacokinetics of cefotaxime. Antimicrob Agents Chemother 1979;16: 592-7.

43.- YUK J H, NIGHTINGALE C H, QUINTILIANI R.
Clinical pharmacokinetics of ceftriaxone. Clin Pharmacokinetic 1989; 17: 223-35.

44.- BRYSKIER A, PROCYK T, LABRO M T. Cefodizime, a new 2-aminothiazolyl cephalosporin: physicochemical properties, toxicology and structureactivity relationships. J Antimicrob Chemother 1990; 26 (Suppl C): 1-8.

45.- CRITCHLEY I A, BASKER M J, EDMONDSON R A, KNOTT S J. Uptake of catecholic cephalosporin by the iron transport system of Escherichia coli. J Antimicrob Chemother 1991; 28: 377-88.

46.- CRITCHLEY I A. Catecholic B-lactams: facilitated transport. J Antimicrob Chemother 1990; 26: 733-7.

47.- JONES R N, ERWIN M E. In vitro evaluation of Ro 09-1227, a novel catechol-substituted cephalosporin. Antimicrob Agents Chemother 1992; 36: 233-8.

48.- JONES R N. In vitro activity of Ro 24-6392, a novel ester-linked co-drug combining ciprofloxacin and desacetylcefotaxime. Eur J Clin Microbiol Infect Dis 1990; 9: 435-8.

49.- TOMASZ A. Penicillin-binding proteins and the antibacterial effectiveness of B-lactam antibiotics. Rev Infect Dis 1986 (Suppl 3); 8: 260-78.

50.- HOLM S E. Interaction between B-lactam and other antibiotics. Rev Infect Dis 1986 (Suppl 3); 8: 305-14.

51.- MC CULLERS J A, ENGLISH B K, NOVAK R. Isolation and characterization of vancomycin-tolerant Streptococcus pneumoniae from the cerebrospinal fluid of a patient who developed recrudescet meningitis. J Infect Dis 2000; 181: 369-73.

52.- NIKAIDO H. Outer membrane barrier as a mechanism of antimicrobial resistance. Antimicrob Agents Chemother 1989; 33: 1831-6.

53.- BARRADELL L B, BRYSON H M. Cefepime. A review of its antibacterial activity, pharmacokinetic properties and therapeutic use. Drugs 1994; 47: 471505.

54.- O'CALLAGHAN C H 1979. Description and classification of the newer cephalosporins and their relationship with the established compounds. J Antimicrob Chemother 5: 635-71

55.- BRYSKIER A, PROCYK T, TREMBLAY D, LENFANT B FOURTILLAN J B. The pharmacokinetics of cefodizime following intravenous and intramuscular administration of a single dose of 1.0 g. J Antimicrob Chemother 1990; 26 (Suppl. C): 59-63.

56.- WILLIAMS J D. Classification of cephalosporins. Drugs. 1987; 34 (Suppl 2): 15-22.

57.- WISEMAN L R, LAMB H M. Cefpirome. A review of its antibacterial activity, pharmacokinetic properties and efficacy in the treatment of severe nosocomial infections and febrile neutropenia. Drugs 1997; 57 : 117-40.

58.- PECHERE J C, WILSON W, NEU H. Laboratory assesment of antibacterial activity of zwitterionic 7methoxyimino cephalosporins. J Antimicrob Chemother 1995; 36: 757-71

59.- GIAMARELLOS-BOURBOULIS EJ, GRECKA P, TSITSIKA A, TYMPANIDOU C, GIAMARELLOU 
H. In-vitro activity of FK 037 (cefoselis), a novel 4 (th) generation cephalosporin, cefepime and cefpirome on nosocomial staphylococci and gram-negative isolates. Diagn Microbiol infect Dis 2000; 36: 18591.

60.- JONES R N, ERWIN M E, BARRETT M S, JOHNSON D M, BRIGGS B M. Antimicrobial activity of E-1040, a novel thiadiazolyl cephalosporin compare with parenteral cephems. Diagn Microbiol infect Dis 1991; 14: 301-9.

61.- IIZAWA Y, OKONOGI K, HAYASHI R, IWAHI T, YAMAZAKI T, IMADA A. Therapeutic effect of cefozopran (SCE-2787), a new parenteral cephalosporin in experimental infections in mice. Antimicrob Agents Chemother 1993; 37: 100-5.

62.- WATANABE N A. Newer antipseudomonal cephalosporins. J Chemother 1996; 8 (Suppl 2): 4856.

63.- ACTOR P, GUARINI J, URI J et al. Disk susceptibility studies with cefazolin and cephalotin. Antimicrob Agents Chemother 1974; 5: 63-7.

64.- UWAYDAH M. Cefazolin in the treatment of acute enteric fever. Antimicrob Agents Chemother 1976; 10: 52-6.

65.- JAMAL W Y, ROTIMI V O, CHUGH T D, PAL T. Prevalence and susceptibility of Shigella to 11 antibiotics in a Kuwait teaching hospital. J Chemother 1998; 10: 285-90.

66.- COX CE, SHERRILL M, COCCHETT DM. Evaluation of cefuroxime axetil, cefaclor, and cephalexin in the treatment of urinary tract infections in adults. Curr Ther Res 1987; 42: 124-37.

67.- SANDERS C W, GREENBERG R N, MARIER R L. Cefamandole and cefoxitin. Ann Intern Med 1985; 103: 70-8.

68.- STAPLEY E P, JACKSON M, HERNANDEZ S et al. Cephamycins, a new family of $\beta$-lactam antibiotics I. Production actinomycetes, including Streptomyces lactamdurans spp.n. Antimicrob Agents Chemother 1972; 2: 122-31

69.- COOPER R D G. The carbacephems a new B-lactams class. Am J Med 1992; 92 (Suppl 6 A): 25-65.

70.- ZEMELMAN R, BELLO H, DOMINGUEZ M, GONZALEZ G, MELLA S, GARCIA A. Activity of imipenem, third-generation cephalosporins, aztreonam, and ciprofloxacin against multi-resistant Gram negative bacilli isolated from Chilean hospitals. J Antimicrob Chemother 1993; 32: 413-9.

71.- MEDEIROS A A. Evolution and dissemination of $\beta$ lactamases accelerated by generation of B-lactam antibiotics. Clin Infect Dis 1997; 24 (Suppl. 1): 1945.

72.- PERRY C M, BROGDEN R N. Cefuroxime axetil: review of its antibacterial activity, pharmacokinetic properties and therapeutic efficacy. Drugs 1996; 52: 125-58.

73.- SCHAAD U B, SUTER S, GIANELLABORRADORI et al. A comparison of ceftriaxone and cefuroxime for the treatment of bacterial meningitis in children. N Engl J Med 1990; 322: 141-7.
74.- KLEIN N C, CUNHA B A. Cefalosporinas de la tercera generación. Clin Med Nort Am 1995; 79: 693 707.

75.- KLUGMAN K P, MADHI S A. Emergence of drug resistance impact on bacterial meningitis. Infect Dis Clin North Am 1999; 13: 637-46.

76.- RICHARDS D M, BROGDEN R N. Ceftazidime. A review of its antibacterial activity, pharmacokinetic properties and therapeutic use. Drugs 1985; 29: 10561.

77.- SCHATZ B S, KARAVOKIROS K T, TAEUBEL M A, ITOKAZU G S. Comparison of cefprozil, cefpodoxime proxetil, loracarbef, cefixime, and ceftibuten. Ann Pharmacother 1996; 30: 258-68.

78.- DALMAU D, LAYRARGUES G P, FENYVES D, WILLEMS $\mathrm{B}$, TURGEON $\mathrm{F}$, TURGEON P. Cefotaxime, desacetyl-cefotaxime, and bactericidal activity in spontaneous bacterial peritonitis. J Infect Dis 1999; 180: 1597-1602.

79.- NIKAIDO W, LIU W, ROSENBERG E Y. Outer membrane permeability and ß-lactamase stability of dipolar ionic cephalosporins containing methoxyimino substituents. Antimicrob Agents Chemother 1990; 34: 337-42.

80.- HANCOCK R E W, BELLIDO F. Factors involved in the enhanced efficacy against gram-negative bacteria of fourth generation cephalosporins. J Antimicrob Chemother 1992; 29(Suppl A): 1-6.

81.- BELLIDO F, PECHERE J C, HANCOCK REW. Novel method for measurement of outer membrane permeability to new beta-lactams in intact Enterobacter cloacae cells. Antimicrob Agents Chemother 1991; 35: 68-72.

82.- NEU H C, CHIN N X, JULES K, LABTHAVIKUL $P$. The activity of BMY-28142, a new broad spectrum beta-lactamase stable cephalosporin. J Antimicrob Chemother 1986; 17: 441-52.

83.- KOBAYASHI S, ARAI S, HAYASHI S, FUJIMOTO K. B-lactamase stability of cefpirome (HR 810), a new cephalosporin with a broad antimicrobial spectrum. Antimicrob Agents Chemother 1986; 30: 713-8.

84.- GARAU J, WILSON W, WOOD M, CARLET J. Fourth generation cephalosporins: a review of in vitro activity, pharmacokinetics, pharmacodynamics, and clinical utility. Clin Microb Infect 1997; 3 (Suppl 1): 87-101.

85.- KESSLER R E, FUNG-TOMC J. Susceptibility of bacterial isolates to beta-lactam antibiotics from U.S. clinical trials over 5-year period. Am J Med 1996; 24 (6 A): 13-9.

86.- PIERARD D, EMMERECHTS K, LAUWERS S. Comparative in-vitro activity of cefpirome against isolates from intensive care and haematology/oncology units. Belgian Multicentric Study Group. J Antimicrob Chemother 1998; 41: 443-50.

87.- JONES R N. Resistance patterns among nosocomial pathogens: trends over the past few years. Chest 2001; 119 (Suppl 2): 397-404.

88.- LORBER B. Changing patterns of infectious diseases. 
Am J Med 1988; 84: 569-78.

89.- MOELLERING R C. A novel antimicrobial agent joins the battle against resistant bacteria. Ann Intern Med 1999; 130: 155-7.
90.- WILSON W R. The role of fourth-generation cephalosporins in the treatment of serious infectious diseases in hospitalized patients. Diagn Microbiol Infect Dis 1998; 31: 473-7.

Correspondencia a:

Sergio Mella Montecinos

Laboratorio de Antibióticos,

Departamento de Microbiología,

Facultad de Ciencias Biológicas

Casilla 160-C, Concepción

E-mail:pignatio@ctcinternet.cl 